\title{
Surface tension of the Widom-Rowlinson model
}

Cite as: J. Chem. Phys. 127, 034707 (2007); https://doi.org/10.1063/1.2751153

Submitted: 03 April 2007. Accepted: 22 May 2007. Published Online: 20 July 2007

E. de Miguel, N. G. Almarza, and G. Jackson

\section{ARTICLES YOU MAY BE INTERESTED IN}

New Model for the Study of Liquid-Vapor Phase Transitions

The Journal of Chemical Physics 52, 1670 (1970); https://doi.org/10.1063/1.1673203

The interfacial tension and phase diagram of the Widom-Rowlinson mixture via Monte Carlo simulations

The Journal of Chemical Physics 128, 014712 (2008); https://doi.org/10.1063/1.2806279

Test-area simulation method for the direct determination of the interfacial tension of systems with continuous or discontinuous potentials

The Journal of Chemical Physics 123, 134703 (2005); https://doi.org/10.1063/1.2038827
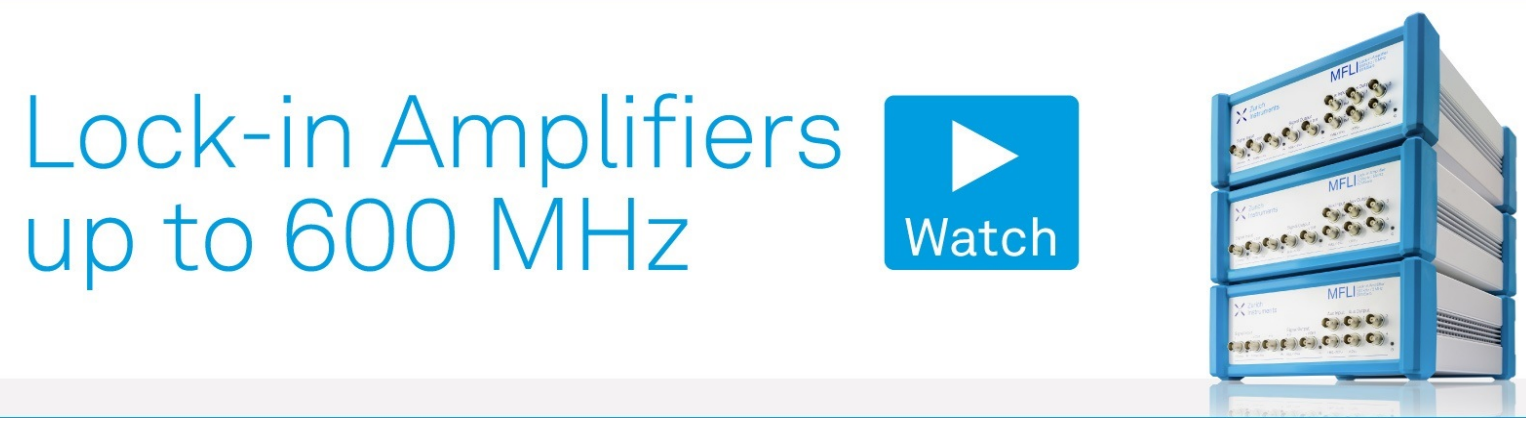

J. Chem. Phys. 127, 034707 (2007); https://doi.org/10.1063/1.2751153

(c) 2007 American Institute of Physics. 


\title{
Surface tension of the Widom-Rowlinson model
}

\author{
E. de Miguel $^{\mathrm{a})}$ \\ Departamento de Física Aplicada, Facultad de Ciencias Experimentales, Universidad de Huelva, \\ 21071 Huelva, Spain
}

N. G. Almarza

Instituto de Química-Física Rocasolano, CSIC, C/Serrano 119, E-28006 Madrid, Spain

G. Jackson

Department of Chemical Engineering, Imperial College London, South Kensington Campus, London SW7 2AZ, United Kingdom

(Received 3 April 2007; accepted 22 May 2007; published online 20 July 2007)

\begin{abstract}
We consider the computation of the surface tension of the fluid-fluid interface for the Widom-Rowlinson [J. Chem. Phys. 52, 1670 (1970)] binary mixture from direct simulation of the inhomogeneous system. We make use of the standard mechanical route, in which the surface tension follows from the computation of the normal and tangential components of the pressure tensor of the system. In addition to the usual approach, which involves simulations of the inhomogeneous system in the canonical ensemble, we also consider the computation of the surface tension in an ensemble where the pressure perpendicular (normal) to the planar interface is kept fixed. Both approaches are seen to provide consistent values of the interfacial tension. The issue of the system-size dependence of the surface tension is addressed. In addition, simulations of the fluid-fluid coexistence properties of the mixture are performed in the semigrand canonical ensemble. Our results are compared with existing data of the Widom-Rowlinson mixture and are also examined in the light of the vapor-liquid equilibrium of the thermodynamically equivalent one-component penetrable sphere model. (C) 2007
\end{abstract} American Institute of Physics. [DOI: 10.1063/1.2751153]

\section{INTRODUCTION}

There is a renewed interest in the description of the statistical mechanics of simple athermal (purely repulsive) models of inhomogeneous mixtures. ${ }^{1,2}$ This stems from a realization that some of these models are able to capture in a simple way the relevant features of the physics that characterize mixtures of sterically stabilized colloids and nonadsorbing polymers. The literature abounds with reports of studies of simple models of both additive and nonadditive mixtures of hard spheres with different approaches. Arguably, one of the simplest athermal models was proposed by Widom and Rowlinson (WR). ${ }^{3}$ The model consists of a symmetric binary mixture in which particles of the same type do not interact, but particles of different type interact via a hardsphere repulsive interaction. The WR model can be considered as a limiting case $(\lambda=0)$ of the family of symmetric $A-B$ nonadditive hard-sphere mixtures ${ }^{4,5}$ defined by hard-sphere interaction diameters $\sigma_{A A}=\sigma_{B B}=\lambda \sigma$ and $\sigma_{A B}=\sigma$, where $\lambda$ is a parameter in the range of $0 \leqslant \lambda \leqslant 1$ for mixtures with positive additivity [i.e., $\left.\left.\sigma_{A B}>\left(\sigma_{A A}+\sigma_{B B}\right) / 2\right)\right]$.

As illustrated in the original paper by Widom and Rowlinson, ${ }^{3}$ one can integrate out the degrees of freedom of one of the components of the WR binary mixture to obtain an effective Hamiltonian of an equivalent one-component model of penetrable spheres characterized by multibody attractive interactions. The isomorphism between the WR and

\footnotetext{
a) Author to whom correspondence should be addressed. Electronic mail: demiguel@uhu.es
}

penetrable sphere models allows one to derive all of the thermodynamic functions of one model from those of the other with simple transcription relations. A trivial implication of the equivalence between the two models is that the vaporliquid equilibrium expected in the one-component penetrable sphere model at low temperatures translates into fluid-fluid demixing of the binary mixture at sufficiently high density. Theoretical approaches to the study of the bulk and interfacial behavior of the WR mixture include the use of the meanfield approximation, ${ }^{3,6-9}$ the virial expansion, ${ }^{10}$ integral equations, ${ }^{11-14}$ and classical density functional theory. ${ }^{15}$ The results of computer simulations of the WR model have been reported, ${ }^{11,12,16-19}$ with a focus on the coexistence properties and critical behavior of the model, apart from the recent note by Malijevský and Sokolowski ${ }^{20}$ who studied the fluid interface of the mixture by Monte Carlo.

The main purpose of this paper is to undertake a thorough computer simulation study of the surface tension of the fluid-fluid interface in the WR binary mixture model. An in-depth analysis of the data in terms of the equivalent onecomponent penetrable sphere model is provided. We examine the WR mixture in the context of simulation data of the interfacial tension for related athermal model mixtures that are also available. ${ }^{21-26}$

There are a number of techniques that can be used to determine the interfacial tension of fluid interfaces (see Ref. 27 and references therein for a recent review on the subject). The usual route involves a mechanical approach ${ }^{6}$ which requires the calculation of the tensorial components of the 
pressure tensor. These are computed during the course of a direct simulation of the inhomogeneous system, typically performed under conditions of fixed number of particles $N$, volume $V$, and temperature $T$. An alternative class of technique $^{28}$ makes use of the relation between the interfacial tension and the probability distribution function of an appropriate order parameter for different sizes of the system. This finite-size scaling approach is arguably more computationally demanding, since it requires accurate computations of the distribution functions under coexistence conditions. $^{22,24,26,29}$ In spite of its complexity and computational cost, techniques based on finite-size scaling are clearly superior in probing the critical region than methods based on the virial (mechanical) route. On the other hand, the latter tends to be more appropriate for states far removed from the critical region, where the free energy barrier that separates the coexisting phases is very high and techniques based on finite-size scaling turn out to be increasingly inefficient. Both approaches are, therefore, quite complementary.

In this paper we make use of the standard mechanical route for the computation of the surface tension of the WR mixture. In addition to the usual approach, which involves a direct simulation of the two coexisting fluids in the canonical $(N V T)$ ensemble (see, for instance, Refs. 30-32), we consider direct simulations of the inhomogeneous system in an ensemble where, in addition to $N$ and $T$, the normal pressure, as well as the area of the (planar) interface, are kept constant. $^{33,34}$ The inputed normal pressure corresponds to the bulk equilibrium pressure of the mixture; this intensive thermodynamic variable can be varied independently along the fluid-fluid coexistence line of the two-component system. The values of the surface tension obtained from simulations at constant volume will be seen to be consistent with those obtained from simulations at constant normal pressure. In Sec. II, we briefly introduce the WR model and show explicitly its equivalence with the one-component penetrable sphere model. We then proceed to examine the coexistence properties of the WR mixture, with particular emphasis on their pressure dependence. The bulk fluid phase equilibria obtained from simulations in the semigrand canonical ensemble ${ }^{35-38}$ is examined in Sec. III, where the vapor-liquid equilibria of the equivalent penetrable sphere model is also considered. The coexistence properties are used to estimate the thermodynamic properties of the WR and penetrable sphere models, and the results are compared with existing data. We then proceed in Sec. IV with the computation of the surface tension of the WR binary mixture. An expression appropriate for the calculation of the surface tension of the mixture at constant (normal) pressure is presented. Our main results are then presented along with an analysis of the size dependence of the surface tension.

\section{THE WIDOW-ROWLINSON MODEL}

In their seminal paper, Widom and Rowlinson ${ }^{3}$ introduced the one-component penetrable sphere model as an offlattice model for the study of the liquid-vapor equilibrium. In the model one assumes that each molecule is surrounded by a sphere of radius $\sigma$ and volume $v_{0}=(4 \pi / 3) \sigma^{3}$. The interac- tions are specified by the configurational energy $U\left(\mathbf{r}^{N}\right)$ of $N$ molecules in a volume $V$ at positions $\mathbf{r}^{N}$, and are given explicitly by

$$
\mathcal{U}\left(\mathbf{r}^{N}\right)=\varepsilon\left[\mathcal{W}\left(\mathbf{r}^{N}\right)-N v_{0}\right] / v_{0},
$$

where $\mathcal{W}\left(\mathbf{r}^{N}\right)$ is the total volume occupied by the $N$ interpenetrating spheres, and $\varepsilon$ is a (positive) parameter that sets the energy scale of the interactions. Any microscopic configuration will satisfy $\mathcal{W}\left(\mathbf{r}^{N}\right) \leqslant N v_{0}$, so that the interactions defined by Eq. (1) are purely attractive; in addition, they are short ranged and are not pairwise additive.

As shown by Widom and Rowlinson, ${ }^{3}$ the onecomponent penetrable sphere model is isomorphous with a symmetric binary mixture (WR model) in which the interactions are purely repulsive and explicitly defined by $\sigma_{A A}$ $=\sigma_{B B}=0$ (there are no interactions between molecules of the same species), and $\sigma_{A B}=\sigma$ (molecules of different species interact as if they were hard spheres of diameter $\sigma$ ). The interactions in the WR model mixture are pairwise additive and symmetric with respect to the exchange of the label of species.

In the following, we examine the explicit equivalence between these two models. Consider the partition function of the system of penetrable spheres in the grand canonical ensemble, ${ }^{39}$

$$
\begin{aligned}
Q(\mu, V, T) & =\sum_{N} \frac{e^{\beta \mu N}}{N ! \Lambda^{3 N}} \int d \mathbf{r}^{N} e^{-\beta \mathcal{U}\left(\mathbf{r}^{N}\right)} \\
& =\sum_{N} \frac{1}{N !}\left(\frac{v_{0}}{\Lambda^{3}}\right)^{N} e^{\beta \mu N} \int \frac{d \mathbf{r}^{N}}{v_{0}^{N}} e^{-\beta \mathcal{U}\left(\mathbf{r}^{N}\right)} \\
& =\sum_{N} \frac{e^{\beta \bar{\mu} N}}{N !} \int \frac{d \mathbf{r}^{N}}{v_{0}^{N}} e^{-\beta \mathcal{U}\left(\mathbf{r}^{N}\right)},
\end{aligned}
$$

where $V$ is the volume, $T$ is the temperature, and $\mu$ is the chemical potential. In the above expression, $\beta=\left(k_{B} T\right)^{-1}$, and a reference chemical potential is defined as $\bar{\mu}=\mu$ $-k_{B} T \ln \left(\Lambda^{3} / v_{0}\right)$, where $\Lambda$ is the de Broglie wavelength, and $k_{B}$ is the Boltzmann constant. Substituting $\mathcal{U}$ from Eq. (1) into Eq. (2), we arrive to

$$
Q(\mu, V, T)=\sum_{N} \frac{e^{\beta(\bar{\mu}+\varepsilon) N}}{N !} \int \frac{d \mathbf{r}^{N}}{v_{0}^{N}} e^{-\beta \varepsilon \mathcal{W}\left(\mathbf{r}^{N}\right) / v_{0}} .
$$

The partition function of the two-component WR mixture in the grand canonical ensemble is given by

$$
\begin{aligned}
Q_{m} & \left(\mu_{A}, \mu_{B}, V, T\right) \\
& =\sum_{N_{A}} \frac{e^{\beta \mu_{A} N_{A}}}{N_{A} ! \Lambda_{A}^{3 N_{A}}} \sum_{N_{B}} \frac{e^{\beta \mu_{B} N_{B}}}{N_{B} ! \Lambda_{B}^{3 N_{B}}} \int d \mathbf{r}^{N_{A}} d \mathbf{r}^{N_{B}} e^{-\beta \mathcal{U}\left(\mathbf{r}^{\left.N_{A}, \mathbf{r}^{N_{B}}\right)}\right.} \\
& =\sum_{N_{A}} \frac{e^{\beta \bar{\mu}_{A} N_{A}}}{N_{A} !} \sum_{N_{B}} \frac{e^{\beta \bar{\mu}_{B} N_{B}}}{N_{B} !} \int \frac{d \mathbf{r}^{N_{A}}}{v_{0}^{N_{A}}} \frac{d \mathbf{r}^{N_{B}}}{v_{0}^{N_{B}}} e^{-\beta \mathcal{U}\left(\mathbf{r}^{N_{A}}, \mathbf{r}^{\left.N_{B}\right)}\right.},
\end{aligned}
$$

where in the second expression the partition function is written in terms of reference chemical potentials such as those defined for the one-component system $\bar{\mu}_{\alpha}=\mu_{\alpha}-k_{B} T \ln \left(\Lambda_{\alpha}^{3} / v_{0}\right)$ with $\alpha=A, B$. The integration and summation with respect to one of the species in the above 
expression can be performed as follows. Consider a given (fixed) configuration $\mathbf{r}^{N_{B}}$ of the (possibly interpenetrating) spheres of type $B$. For any one of the $N_{A}$ particles, with coordinates $\mathbf{r}_{A}$, the integral $\int d \mathbf{r}_{A} \exp \left\{-\beta\left[\mathcal{U}\left(\mathbf{r}^{N_{A}}, \mathbf{r}^{N_{B}}\right)\right]\right\}$ represents the free volume accessible to this particle due to the presence of the spheres of type $B$. This is simply given by $V-\mathcal{W}\left(\mathbf{r}^{N_{B}}\right)$, where the function $\mathcal{W}$ has exactly the same meaning as the function $\mathcal{W}$ introduced earlier in the context of the penetrable sphere model. As the particles of the same type do not interact with each other the integral over all particles of type $A$ will yield an equivalent free volume, so that one can write

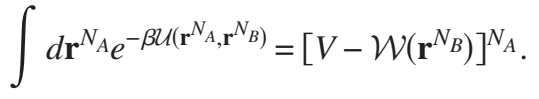

On substituting this result back into Eq. (4), the grand canonical partition function of the WR binary mixture can be expressed as

$$
\begin{aligned}
Q_{m}\left(\mu_{A}, \mu_{B}, V, T\right)= & \sum_{N_{B}} \frac{e^{\beta \bar{\mu}_{B} N_{B}}}{N_{B} !} \int \frac{d \mathbf{r}^{N_{B}}}{v_{0}^{N_{B}}} \sum_{N_{A}} \frac{1}{N_{A} !} \\
& \left.\times\left[\frac{\bar{z}_{A}\left[V-\mathcal{W}\left(\mathbf{r}^{N_{B}}\right)\right]}{v_{0}}\right]\right]^{N_{A}},
\end{aligned}
$$

where we have defined the activity of component $A$ as $\bar{z}_{A}=\exp \left(\beta \bar{\mu}_{A}\right)$. The sum with respect to component $A$ can be readily performed by noting that $\Sigma_{n} x^{n} / n !=\exp (x)$ to give

$$
\begin{aligned}
Q_{m}\left(\mu_{A}, \mu_{B}, V, T\right) & =\sum_{N_{B}} \frac{e^{\beta \bar{\mu}_{B} N_{B}}}{N_{B} !} \int \frac{d \mathbf{r}^{N_{B}}}{v_{0}^{N_{B}}} e^{-\bar{z}_{A} V / v_{0}} e^{-\bar{z}_{A} \mathcal{W}\left(\mathbf{r}^{\left.N_{B}\right) / v_{0}}\right.} \\
& =e^{\bar{z}_{A} V / v_{0}} \sum_{N_{B}} \frac{e^{\beta \bar{\mu}_{B} N_{B}}}{N_{B} !} \int \frac{d \mathbf{r}^{N_{B}}}{v_{0}^{N_{B}}} e^{-\bar{z}_{A} \mathcal{W}\left(\mathbf{r}_{B}^{\left.N_{B}\right) / v_{0}} .\right.}
\end{aligned}
$$

A comparison between Eqs. (3) and (7) allows one to establish the following transcription relations between both models:

$$
\begin{aligned}
& N=N_{B}, \\
& \beta(\bar{\mu}+\varepsilon)=\beta \bar{\mu}_{B}, \\
& \beta \varepsilon=\bar{z}_{A},
\end{aligned}
$$

where the magnitudes on the left-hand side refer to the onecomponent system, while those on the right-hand side correspond to the binary mixture. From Eqs. (3) and (7), and using Eqs. (8)-(10), it follows that the partition functions of the thermodynamically isomorphous systems satisfy the relation

$$
\ln Q_{m}=\ln Q+\frac{\bar{z}_{A} V}{v_{0}} .
$$

Considering that the pressure in the grand canonical ensemble is given by $\beta P=(\partial \ln Q / \partial V)_{\mu V T}$ (one component) and $\beta P_{m}=\left(\partial \ln Q_{m} / \partial V\right)_{\mu_{A} \mu_{B} V T}$ (mixture), expression (11) leads to the following relation between the pressures in both systems:

$$
\beta P=\beta P_{m}-\frac{\bar{z}_{A}}{v_{0}} .
$$

For an inhomogeneous one-component system with an interface of area $A$, the corresponding surface tension $\gamma$ follows from $\gamma=(\partial \Omega / \partial A)_{\mu V T}$, where $\Omega=-k_{B} T \ln Q$ is the grand potential. ${ }^{6}$ Similarly, the interfacial tension in a binary mixture follows from $\gamma_{m}=\left(\partial \Omega_{m} / \partial A\right)_{\mu_{A} \mu_{B} V T}$. From the transcription relation (11), it is straightforward to show that the surface tension of the vapor-liquid interface in the penetrable sphere model $\gamma$ and the surface tension of the fluid-fluid interface in the WR mixture $\gamma_{m}$ satisfies $\gamma=\gamma_{m}$. In the appropriate reduced units, the interfacial tension of the penetrable sphere model can be readily obtained from the value of the reduced interfacial tension of the WR mixture as

$$
\gamma^{*}=T^{*} \gamma_{m}^{*},
$$

where $\gamma^{*}=\gamma \sigma^{2} / \varepsilon, \gamma_{m}^{*}=\gamma_{m} \sigma^{2} \beta$, and $T^{*}=k_{B} T / \varepsilon$ is the temperature at which vapor-liquid separation takes place in the one-component model. According to Eq. (10), the reduced temperature $T^{*}$ is related to the activity of component $A$ through $T^{*}=1 / \bar{z}_{A}$.

\section{COEXISTENCE PROPERTIES}

In this section we present simulation results for the fluidfluid coexistence properties of the WR mixture and the corresponding vapor-liquid properties of the penetrable sphere model. Monte Carlo simulations are performed in the semigrand canonical ensemble, ${ }^{35}$ where the total number of particles of the mixture $N=N_{A}+N_{B}$, and the difference $\Delta \mu$ of the chemical potential of species $A$ and $B$ are kept fixed. The composition of the mixture is allowed to fluctuate by performing identity changes at constant volume (or pressure) and at constant $\Delta \mu$. Owing to the symmetry of the WR mixture, the chemical potentials of species $A$ and $B$ are equal in each phase under coexistence conditions, so that setting $\Delta \mu=0$ yields the demixing line provided that the resulting average compositions are different. ${ }^{36-38}$ The pressure of the mixture is calculated from the virial route, which for the WR mixture allows one to express the pressure in terms of the number of $A-B$ pairs $N_{A B}$ that overlap under a virtual (test) compression of the system. ${ }^{40-44}$ In the case of the semigrand ensemble we can write [cf. Eq. (20) of Ref. 44]

$$
\beta P=\lim _{\Delta V \rightarrow 0}\left\langle\rho-\frac{1}{\Delta V} N_{A B}\right\rangle,
$$

where $\rho=N / V$ is the total number density, $\Delta V$ is the magnitude of the virtual volume change, and where the angular brackets indicate an ensemble average. In practice, the pressure is calculated by computing $N_{A B}$ for a set of values of $\Delta V$ and an extrapolation to $\Delta V \rightarrow 0$ is then undertaken. The chemical potential of one of the species (here taken to be of type $A$ ) is calculated with the Widom insertion method ${ }^{45}$ using $10^{4}$ trial insertions of particles of type $A$ per cycle.

Once the mixture is equilibrated at the input conditions, averages are collected over $5 \times 10^{5}$ cycles, where each cycle consists of $N$ trial molecular displacements, and 200 to 1000 attempts to perform identity changes $A \rightarrow B$ or $B \rightarrow A$ with 


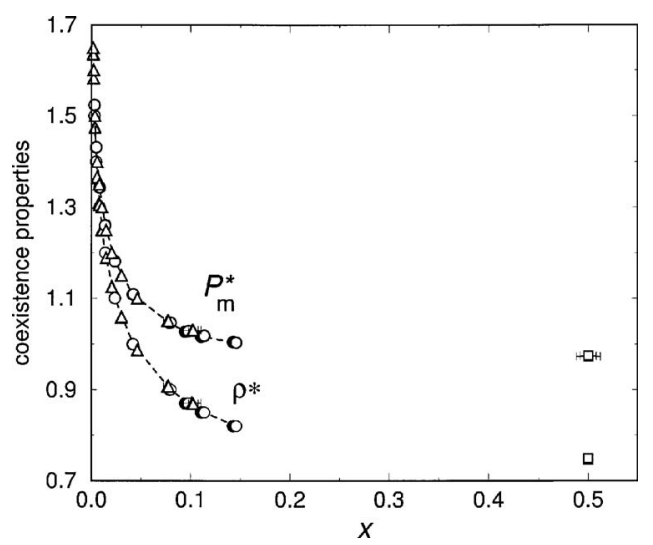

FIG. 1. The fluid-fluid coexistence data of the Widom-Rowlinson (WR) mixture in the density-composition and pressure-composition planes as obtained from simulations in the semigrand canonical ensemble at constant volume (circles) and at constant pressure (triangles) for systems of $N=864$ (filled symbols) and $N=2000$ (open symbols) particles. We show the total reduced density $\rho^{*}=\rho \sigma^{3}$ of one of the coexisting phases and the reduced pressure $P_{m}^{*}=\beta P \sigma^{3}$. The squares represent the critical pressure and density (critical composition $x_{c}=0.5$ ).

equal probability (the precise number depending on the input thermodynamic conditions). In order to avoid serial correlations, averages and estimates of the errors are calculated by dividing the simulation run into $M=50$ blocks. An estimate of the statistical precision of the sample averages is given by the standard deviation in the block averages calculated from $\sigma / \sqrt{M}$, where $\sigma$ is the variance of the block averages and $M$ is the total number of blocks. ${ }^{46}$ The simulations correspond to the $A$-rich phase and are performed for systems containing a total of $N=864$ and 2000 molecules.

The demixing region of the WR mixture in the total density-composition and pressure-composition $\left(x=x_{B}\right)$ representations for the two system sizes considered is shown in Fig. 1. Owing to the symmetry of the WR mixture, the critical composition is $x_{c}=0.5$, the coexistence curve being symmetric about this axis (only the portion corresponding to $x<0.5$ is shown in the figure). The coexistence properties are seen to be insensitive to whether the simulations are performed at constant volume or at constant pressure, the only difference being that larger fluctuations in the averages are generally observed under constant-pressure conditions. The results are found to be fully consistent with those previously reported. ${ }^{5,12}$ The bulk coexistence properties are not seen to exhibit any significant dependence with $N$ in the range of thermodynamic conditions considered here. System-size effects, however, are expected to become significant as the system approaches the critical point.

We show in Fig. 2 the fluid-fluid coexistence region of the WR mixture in the activity-density plane, where a residual activity of species $A$ is defined as $z_{A}^{*}=\exp \left(\beta \tilde{\mu}_{A}\right)$, with $\tilde{\mu}=\mu-k_{B} T \ln \left(\Lambda^{3} / \sigma^{3}\right)$ being the relative chemical potential, and where $\rho_{A} \sigma^{3}$ is the density of component $A$ in the coexisting fluid phases I and II. The corresponding vapor-liquid coexistence curve of the equivalent one-component penetrable sphere model is also shown in the figure. From the transcription relations (8)-(10), the liquid $\left(\rho_{l}\right)$ and vapor $\left(\rho_{v}\right)$
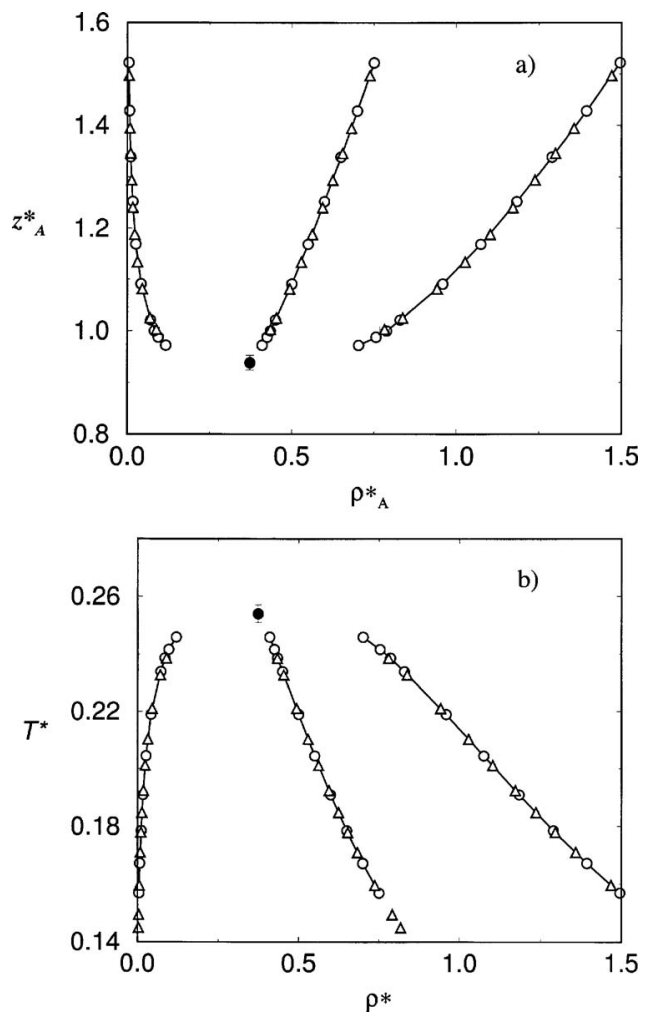

FIG. 2. (a) The fluid-fluid coexistence curve and curve of rectilinear diameters of the WR mixture in the activity-density plane as obtained from simulations in the semigrand canonical ensemble at constant volume (circles) and constant pressure (triangles) for systems with $N=2000$ particles. The relative activity of species $A$ is defined as $z_{A}^{*}=\exp \left(\beta \tilde{\mu}_{A}\right)$, where $\tilde{\mu}=\mu$ $-k_{B} T \ln \left(\Lambda^{3} / \sigma^{3}\right)$ is the reduced chemical potential, and $\rho_{A}^{*}=\rho_{A} \sigma^{3}$ is the density of component $A$ in the coexisting fluid phases. The filled symbol locates the critical point. (b) The corresponding vapor-liquid coexistence curve of the equivalent one-component penetrable sphere model. The reduced temperature is defined as $T^{*}=k_{B} T / \varepsilon$. The filled symbol represents the critical point. The continuous curves are drawn to guide the eyes.

densities are given by $\rho_{l}=\rho_{A}^{\mathrm{I}}$ and $\rho_{v}=\rho_{A}^{\mathrm{II}}$, and the reduced temperature $T^{*}$ follows from Eq. (10), where $\bar{z}_{A}=\left(v_{0} / \sigma^{3}\right) z_{A}^{*}$ with $v_{0}=(4 \pi / 3) \sigma^{3}$.

The variation of the pressure of the WR mixture $P_{m}^{*}=\beta P_{m} \sigma^{3}$ along the fluid-fluid transition line is depicted in Fig. 3 in terms of the total density of the mixture $\rho^{*}$ and the activity $z_{A}^{*}$. As expected, $P_{m}^{*}$ tends assymptotically to $\rho^{*}$ and $z_{A}^{*}$ in the high-density regime, where the system is expected to behave essentially as two immiscible ideal gases.

Though we did not probe the critical region, we use the coexistence data obtained from simulations to estimate the thermodynamic properties at the fluid-fluid (vapor-liquid) critical point. The corresponding assymptotic behavior of the order parameter and the diameter of the vapor-liquid coexistence curve pertaining to the one-component penetrable sphere model are known to be given by $\left(\rho_{l}-\rho_{v}\right) \propto|t|^{\beta}$ and $\left(\rho_{l}+\rho_{v}\right) / 2-\rho_{c} \propto|t|^{1-\alpha}$, respectively, where $t=1-T / T_{c}$ is the relative distance from the critical temperature $T_{c}, \rho_{c}$ is the value of the density at the critical point, and $\alpha$ and $\beta$ are the corresponding critical exponents. The exponent $\beta$ is not to be confused with inverse temperature. From relation (10), it follows that an appropriate thermodynamic field for the athermal WR mixture is the activity $\tilde{z}=z / z_{c}-1$ of one of the components of the mixture, where $\tilde{z}$ is the relative distance from 

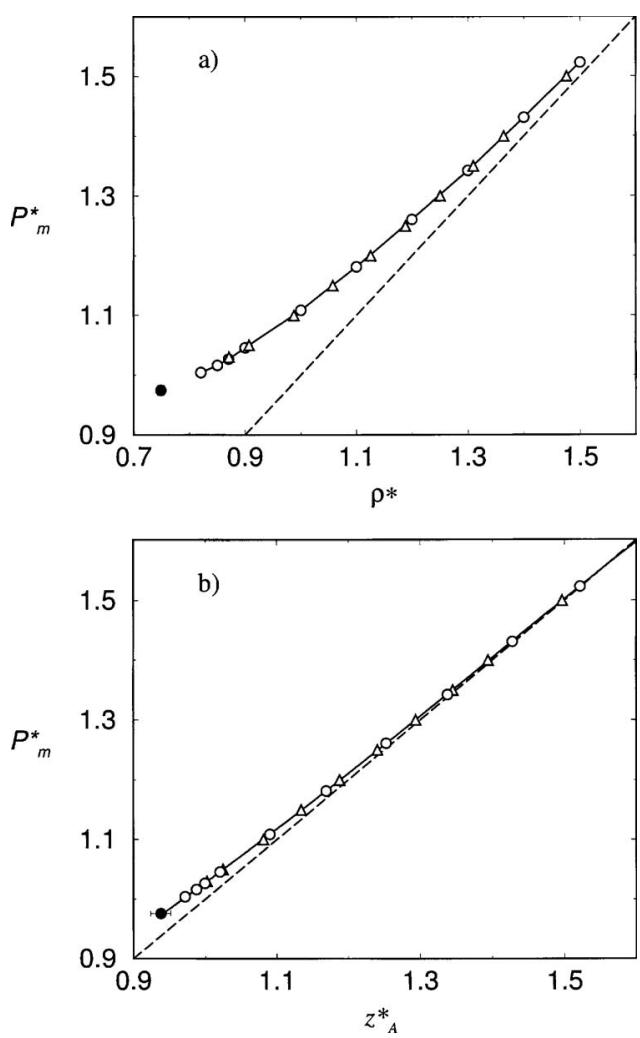

FIG. 3. The fluid-fluid coexistence curve of the WR mixture in the (a) pressure-density and (b) pressure-activity planes as obtained from simulations in the semigrand canonical ensemble at constant volume (circles) and constant pressure (triangles) for systems of $N=2000$ particles. The filled symbols represent the critical point. The continuous curve is drawn to guide the eyes and the dashed line represents the expected ideal-gas behavior at high pressure.

the critical activity $z_{c}$. Using expressions (8)-(10) the appropriate assymptotic relations for the athermal WR mixture can be summarized as

$$
\begin{aligned}
& \left.\left(\rho_{A}-\rho_{B}\right) \propto|z|\right|^{\beta}, \quad\left(\rho-\rho_{c}\right) \propto|\bar{z}|^{1-\alpha}, \\
& \left(\rho_{A}-\rho_{B}\right) \propto\left(\rho-\rho_{c}\right)^{\beta /(1-\alpha)},
\end{aligned}
$$

where $\rho=\rho_{A}+\rho_{B}$ is the total density, and $\rho_{c}$ is its value at the critical point. Only the eight points closest to the critical point are considered to estimate the critical properties of the WR mixture; for this calculation, we make use of Eq. (15) with $\beta=0.324$ and $\alpha=0.113{ }^{26}$ The resulting estimates for the two system sizes considered here are included in Table I, along with the critical properties of the penetrable sphere model that follow from the transcription relations. Our estimates of the critical properties of the WR mixture are consistent with those obtained from the more accurate finite-size scaling (FSS) technique. The values obtained here for the critical activity, $z_{c}^{*}=0.938 \pm 0.014(N=864)$ and $z_{c}^{*}=0.940 \pm 0.009(N=2000)$, are to be compared with the FSS value $z_{c}^{*} \approx 0.9403$ (Ref. 16) and $0.93791 \pm 0.00004 .{ }^{19}$ We estimate the critical density to be $\rho_{c}^{*}=0.746 \pm 0.004(N=864)$ and $\rho_{c}^{*}=0.749 \pm 0.003(N=2000)$, while the reported values are $\quad 0.762 \pm 0.016,{ }^{12} \quad 0.748 \pm 0.002,{ }^{16} \quad 0.759 \pm 0.019,{ }^{5}$ $0.7470 \pm 0.0008,{ }^{17}$ and $0.7486 \pm 0.0002 .{ }^{19}$

In order to obtain an estimate of the critical pressure of the mixture we first fit the values of the pressure obtained from simulations along the demixing line to a virial-like expression of the form $P_{m}^{*}\left(z_{A}^{*}\right)=\sum_{n=1}^{5} a_{n}\left(z_{A}^{*}\right)^{n}$ with $a_{1}=1$, and extrapolate to the critical point, i.e., $\left(P_{m}^{*}\right)_{c}=P_{m}^{*}\left(z_{c}^{*}\right)$. Here, $z_{c}^{*}$ is the critical activity obtained as explained earlier. We find $\left(P_{m}^{*}\right)_{c}=0.973 \pm 0.012(N=864) \quad$ and $\quad\left(P_{m}^{*}\right)_{c}=0.975 \pm 0.008$ $(N=2000)$. Using the transcription relation (12), one finds $P_{c}^{*}=0.009 \pm 0.005(N=864)$ and $P_{c}^{*}=0.009 \pm 0.003(N=2000)$ for the critical pressure of the one-component fluid of penetrable spheres, where $P^{*}=P \sigma^{3} / \varepsilon$. These values are fully consistent with those that would be obtained from an extrapolation of the saturated vapor pressures using the Clausius-Clapeyron equation. These values are to be compared with those obtained by Widom and Rowlinson ${ }^{3}$ for the vapor-liquid critical point $\rho_{c}^{*}=1 / v_{0}=0.2387, \quad T_{c}^{*}=1 / e$ $=0.3679$, and $P_{c}^{*}=(3 / e-1) / v_{0}=0.0247$ with a mean-field theory. The corresponding values for the WR mixture within this approximation are $\rho_{c}^{*}=2 / v_{0}=0.4775, z_{c}^{*}=e / v_{0}=0.6489$, and $\left(P_{m}^{*}\right)_{c}=3 / v_{0}=0.7162$.

\section{SURFACE TENSION}

The computation of the surface tension of the WR binary mixture is now considered by calculation of the pressure tensor in constant-volume or constant-pressure simulations of the inhomogeneous system formed from two coexisting fluid phases separated by an interface.

For a planar interface lying in the $x-y$ plane, mechanical arguments ${ }^{6}$ allow the surface tension $\gamma$ to be expressed in terms of the local components of the pressure tensor as $\gamma=\int_{-\infty}^{\infty} d z\left[P_{N}(z)-P_{T}(z)\right]$, where $P_{N}(z)$ and $P_{T}(z)$ are the normal and tangential components, respectively, and $z$ is the distance from the interface. At equilibrium, $P_{N}(z)$ does not

TABLE I. The fluid-fluid critical properties of the Widom-Rowlinson (WR) mixture and the corresponding estimates of the vapor-liquid critical properties of the one-component penetrable sphere model estimated from simulation in the semigrand canonical ensemble for systems containing $N$ particles. The reduced activity of one of the species, total number density, and pressure of the WR mixture are defined as $z^{*}=\exp (\beta \tilde{\mu})$, where $\tilde{\mu}=\mu-k_{B} T \ln \left(\Lambda^{3} / \sigma^{3}\right), \rho^{*}=\rho \sigma^{3}$, and $P_{m}^{*}=\beta P_{m} \sigma^{3}$, respectively. The reduced temperature, number density, and pressure of the penetrable sphere model are defined as $T^{*}=k_{B} T / \varepsilon, \rho^{*}=\rho \sigma^{3}$, and

\begin{tabular}{|c|c|c|c|c|c|c|}
\hline \multirow[b]{2}{*}{$N$} & \multicolumn{3}{|c|}{ WR mixture } & \multicolumn{3}{|c|}{ Penetrable sphere model } \\
\hline & $z_{c}^{*}$ & $\rho_{c}^{*}$ & $\left(P_{m}^{*}\right)_{c}$ & $T_{c}$ & $\rho_{c}^{*}$ & $P_{c}^{*}$ \\
\hline 864 & $0.938(14)$ & $0.7456(44)$ & $0.973(12)$ & $0.255(4)$ & $0.3728(22)$ & $0.009(5)$ \\
\hline 2000 & $0.940(9)$ & $0.7487(28)$ & $0.975(8)$ & $0.254(3)$ & $0.3744(14)$ & $0.009(3)$ \\
\hline MF & 0.6489 & 0.4775 & 0.7162 & 0.3679 & 0.2387 & 0.0247 \\
\hline
\end{tabular}
$P^{*}=P \sigma^{3} / \varepsilon$, respectively. The last row includes the corresponding predictions form a mean-field approximation (Ref. 3). 
depend on $z$ and corresponds to the equilibrium pressure $P$. Thermodynamic arguments ${ }^{47}$ can be invoked to show that $\gamma=L_{z}\left(P_{N}-P_{T}\right)$, where the macroscopic averages of the tensorial components $P_{N}$ and $P_{T}$ can be defined in terms of appropriate derivatives of the Helmholtz free energy $F$ as

$$
P_{N}=-\left(\frac{\partial F}{\partial V}\right)_{N, T, A}, \quad P_{T}=-\left(\frac{\partial F}{\partial V}\right)_{N, T, L_{z}},
$$

where $L_{z}$ is the longitudinal dimension of the system, and $A=L_{x} L_{y}$ is the area of the planar interface. In a constantvolume simulation, $L_{z}$ is fixed and $\gamma$ follows from the computation of the average values of $P_{N}$ and $P_{T}$ from virtual volume changes within the mechanical approach.

In the case of binary systems, one can also consider an ensemble characterized by a fixed value of the interfacial area $A$ and constant normal pressure $P_{N}$ (equal to the pressure of the mixture at coexistence), so that the longitudinal dimension of the system $L_{z}$ is a fluctuating quantity in the course of the simulation. The surface tension can be computed in this case as an appropriate average over the $N P_{N} A T$ ensemble, the explicit expression being ${ }^{33,34}$

$$
\gamma=\left\langle L_{z}\left[P_{N}-P_{T}(V)\right]\right\rangle,
$$

where $P_{T}(V)$ denotes the tangential pressure of a configuration of the system of volume $V=A L_{z}$. A derivation of this expression can be obtained by considering the Gibbs free energy $G$. For a system at constant applied pressure $P$, the surface tension is defined as the thermodynamic derivative of the Gibbs free energy with area,

$$
\gamma=\left(\frac{\partial G}{\partial A}\right)_{N, P, T}=-k_{B} T \frac{1}{Q}\left(\frac{\partial Q}{\partial A}\right)_{N, P, T},
$$

where $Q$ is the partition function of the system, ${ }^{48}$

$$
Q \equiv Q(N, P, A, T)=\frac{1}{V_{0}} \int d V \exp \{-\beta[P V+F(V)]\} .
$$

Here, $V_{0}$ is an arbitrary unit of volume that renders the partition function dimensionless. In the above expression, $F(V)$ is the Helmholtz free energy of a configuration of the system with volume $V$, where $V=A L_{z}$. On differentiating Eq. (19) with respect to the interfacial area $A$, and recalling that $P_{N}$ $=P$, we obtain

$$
\begin{aligned}
\gamma= & -\frac{k_{B} T}{V_{0}} \frac{1}{Q} \int d V(-\beta)\left[P_{N} L_{z}+\left(\frac{\partial F(V)}{\partial A}\right)_{L_{z}}\right] \\
& \times \exp \left\{-\beta\left[P_{N} V+F(V)\right]\right\} \\
= & \left\langle P_{N} L_{z}+\left(\frac{\partial F(V)}{\partial A}\right)_{L_{z}}\right\rangle_{N, P_{N}, A, T},
\end{aligned}
$$

where the angular brackets denote an average over the $N P_{N} A T$ ensemble. According to Eq. (16),

$$
\left(\frac{\partial F(V)}{\partial A}\right)_{L_{z}}=L_{z}\left(\frac{\partial F(V)}{\partial V}\right)_{L_{z}}=-L_{z} P_{T}(V) .
$$

Expression (17) follows after substitution of Eq. (21) into Eq. (20). We recall that in Eq. (17) $P_{N}$ corresponds to the (input) normal pressure, $P_{T}(V)$ to the (fluctuating) tangential component of the pressure tensor of a configuration of the system with volume $V$, and $L_{z}$ to the (fluctuating) normal dimension of the simulation cell.

All of the simulations for inhomogeneous systems reported here are performed for an equimolar WR mixture. In all cases, the simulation cell consists of an elongated box of dimensions $L_{x}=L_{y} \equiv L$ and $L_{z}>L$. In general, the initial configuration is generated by placing $N_{A}$ molecules of type $A$ with random coordinates within the region defined by $0 \leqslant x_{i}, y_{i} \leqslant L_{x}$ and $\sigma / 2 \leqslant z_{i} \leqslant L_{z} / 2-\sigma / 2\left(i=1, \ldots, N_{A}\right)$ where $\sigma$ is the molecular diameter. This defines the $A$-rich phase. Similarly, the $B$-rich phase is generated by placing $N_{B}$ molecules of type $B$ with random coordinates within the region defined by $0 \leqslant x_{i}, \quad y_{i} \leqslant L_{x}$ and $L_{z} / 2+\sigma / 2 \leqslant z_{i} \leqslant L_{z}-\sigma / 2$ $\left(i=1, \ldots, N_{B}\right)$. By employing standard periodic boundary conditions in all three directions, this setup stabilizes two planar interfaces perpendicular to the $z$ direction under suitable thermodynamic (coexistence) conditions. Some simulations are also initialized from the final configuration of a previously equilibrated simulation at different thermodynamic conditions to speed up the equilibration process. The configurational space is sampled by considering the usual Metropolis Monte Carlo scheme. Diffusion of the particles throughout the system is achieved by performing particle displacements and identity swaps, ${ }^{37,38}$ so that the overall composition of the mixture remains fixed.

The simulations are performed in cycles, where 1 cycle consists of $N$ attempts to displace the molecules and between 250 to 500 identity swaps. Owing to the particular nature of the interactions of the WR mixture, we find that the configuration space is also appropriately sampled if rather than swaps of particle identity one considers translational moves, whereby the trial position of the particles is selected at random within the simulation cell. Both schemes are found to yield equivalent results. Simulations performed at constant pressure include an additional step in which the volume of the system is changed by varying the longitudinal dimension of the box $L_{z}$ while keeping the transverse (interfacial) area constant. The simulations are carried out for systems of $N=1500$ and 4000 particles. Once the system is equilibrated, averages are collected over a total of $10^{6}$ cycles (for $N=1500$ ) or $4 \times 10^{6}$ cycles (for $N=4000$ ). The simulation is divided into blocks of length $5 \times 10^{4}$ cycles and ensemble averages are computed from the arithmetic mean of the corresponding coarse-grained (or block) averages. An estimate of the errors is obtained as explained earlier.

In the constant-volume simulations, the dimensions of the box are chosen such that $L_{z}=3 L_{x}$ and the surface tension is calculated from

$$
\gamma=\frac{1}{2}\left\langle L_{z}\left(P_{N}-P_{T}\right)\right\rangle,
$$

where the prefactor accounts for the existence of two fluidfluid interfaces in the system. 
TABLE II. Values of the reduced pressure $P_{m}^{*}=\beta P_{m} \sigma^{3}$ and fluid-fluid surface tension $\gamma_{m}^{*}=\beta \gamma_{m} \sigma^{2}$ of the Widom-Rowlinson mixture obtained from simulations of the inhomogeneous fluid-fluid system at constant volume with $N=1500$ and $N=4000$ particles. The dimension of the simulation box are $L_{x}=L_{y}=L$ and $L_{z}=3 L$. The error bars correspond to twice the value of the standard deviation of the block averages. $\gamma^{*}=\gamma \sigma^{2} / \varepsilon$ is the vapor-liquid surface tension of the isomorphous one-component penetrable sphere model.

\begin{tabular}{|c|c|c|c|c|c|c|c|}
\hline \multicolumn{4}{|c|}{$N=1500$} & \multicolumn{4}{|c|}{$N=4000$} \\
\hline$L / \sigma$ & $P_{m}^{*}$ & $\gamma_{m}^{*}$ & $\gamma^{*}$ & $L / \sigma$ & $P_{m}^{*}$ & $\gamma_{m}^{*}$ & $\gamma^{*}$ \\
\hline 8.480 & $1.0270(11)$ & $0.044(4)$ & $0.0105(10)$ & 11.759 & $1.0203(2)$ & $0.036(4)$ & $0.0087(14)$ \\
\hline 8.314 & $1.0592(10)$ & $0.070(5)$ & $0.0161(12)$ & 11.529 & $1.0511(5)$ & $0.056(4)$ & $0.0130(9)$ \\
\hline 8.221 & $1.0824(14)$ & $0.086(4)$ & $0.0194(9)$ & 11.400 & $1.0710(3)$ & $0.072(5)$ & $0.0164(11)$ \\
\hline 7.937 & $1.1577(8)$ & $0.144(4)$ & $0.0301(8)$ & 11.006 & $1.1438(3)$ & $0.129(4)$ & $0.0273(8)$ \\
\hline 7.689 & $1.2432(10)$ & $0.217(3)$ & $0.0420(6)$ & 10.662 & $1.2252(3)$ & $0.196(3)$ & $0.0385(6)$ \\
\hline 7.469 & $1.3353(9)$ & $0.287(3)$ & $0.0515(5)$ & 10.357 & $1.3134(2)$ & $0.268(3)$ & $0.0490(5)$ \\
\hline 7.368 & $1.3835(6)$ & $0.330(4)$ & $0.0571(7)$ & 10.217 & $1.3594(2)$ & $0.303(4)$ & $0.0534(7)$ \\
\hline 7.272 & $1.4324(6)$ & $0.369(3)$ & $0.0617(5)$ & 10.085 & $1.4066(5)$ & $0.340(3)$ & $0.0579(5)$ \\
\hline 7.095 & $1.5338(6)$ & $0.451(3)$ & $0.0703(5)$ & 9.839 & $1.5037(2)$ & $0.419(3)$ & $0.0667(5)$ \\
\hline 6.934 & $1.6382(5)$ & $0.532(3)$ & $0.0776(4)$ & 9.615 & $1.6042(2)$ & $0.496(3)$ & $0.0740(4)$ \\
\hline
\end{tabular}

The normal component of the pressure tensor $P_{N}$ is computed with Eq. (14), with $N_{A B}$ representing the number of $A-B$ molecular pairs that overlap after a fictitious (test) volume perturbation in which $L_{z}$ is changed to $L_{z}-\Delta L_{z}$ at constant transverse area $A$. A similar procedure is followed for the calculation of the tangential component $P_{T}$, where the volume change involves now an isotropic change of the cross-section area of the box $A$ to $A-\Delta A$ at constant $L_{z}$. In the constant-pressure simulations, the surface tension is computed using a similar expression, the only difference being that $P_{N}=P$ is now fixed and corresponds to the external pressure, and $L_{z}$ is a fluctuating quantity. As a check of consistency, the average value of $P_{N}$ is also calculated by determining the number of overlaps when the volume is changed. In all cases, this average is found to be equal to the prescribed input pressure within statistical uncertainties. The components of the pressure tensor and the surface tension are calculated every two cycles for the set of five values of the volume change defined by $\Delta V / V=-0.0025 k$, with $k=1, \ldots, 5$, and the instantaneous value is obtained from a linear extrapolation to $\Delta V / V \rightarrow 0$.

The resulting values of the interfacial tension of the WR mixture are included in Tables II (constant-volume simula- tions) and III (constant-pressure simulations). Statistical uncertainties are generally found to be larger for the constantpressure simulations, where the volume of the system is allowed to fluctuate. The computed values of the interfacial tension are plotted in Fig. 4 in terms of the pressure of the mixture. For the smallest systems of $N=1500$ particles, the values of the surface tension obtained in the constant-volume simulations [cf. Fig. 4(a)] are seen to be slightly, but systematically, smaller than those obtained from simulations at constant pressure over the whole range of pressures considered here. These differences are believed to be due to system-size effects, which are expected to become more noticeable for interfacial properties in comparison to what is found for the bulk coexistence properties. This is corroborated by the fact that the differences become vanishingly small when systems containing $N=4000$ particles are considered, as can be appreciated from Fig. 4(b). One should bear in mind that the main contributions to the surface tension arise from the small spacial region over which the surface extends. These contributions are thus expected to depend on the interfacial area $A$. While the same value of $A$ is used in our constant-pressure simulations, different values of $A$ are employed in the series of simulations performed at constant volume.

TABLE III. Values of the reduced fluid-fluid surface tension $\gamma_{m}^{*}=\beta \gamma_{m} \sigma^{2}$ of the Widom-Rowlinson mixture obtained from simulations of the inhomogeneous fluid-fluid system at constant pressure $P_{m}^{*}=\beta P_{m} \sigma^{3}$ (which also corresponds to the normal component of the pressure tensor) and constant interfacial area $A$ $=L \times L$ for systems containing $N=1500(L=7.368 \sigma)$ and $N=4000(L=10 \sigma)$ particles. $L_{z}$ corresponds to the ensemble average value of the longitudinal dimension of the simulation cell. The error bars correspond to twice the value of the standard deviation of the block averages. $\gamma^{*}=\gamma \sigma^{2} / \varepsilon$ is the vapor-liquid surface tension of the isomorphous one-component penetrable sphere model.

\begin{tabular}{|c|c|c|c|c|c|c|}
\hline \multirow[b]{2}{*}{$P_{m}^{*}$} & \multicolumn{3}{|c|}{$N=1500$} & \multicolumn{3}{|c|}{$N=4000$} \\
\hline & $L_{z} / \sigma$ & $\gamma_{m}^{*}$ & $\gamma^{*}$ & $L_{z} / \sigma$ & $\gamma_{m}^{*}$ & $\gamma^{*}$ \\
\hline 1.05 & $31.94(8)$ & $0.080(11)$ & $0.019(3)$ & $45.60(8)$ & $0.062(22)$ & $0.014(5)$ \\
\hline 1.10 & $29.58(4)$ & $0.117(8)$ & $0.0259(18)$ & $42.12(4)$ & $0.106(13)$ & $0.023(3)$ \\
\hline 1.20 & $25.159(16)$ & $0.193(6)$ & $0.0388(12)$ & $37.155(17)$ & $0.184(9)$ & $0.0370(18)$ \\
\hline 1.30 & $23.704(14)$ & $0.273(5)$ & $0.0504(9)$ & $33.637(18)$ & $0.263(8)$ & $0.0486(15)$ \\
\hline 1.40 & $21.834(14)$ & $0.352(4)$ & $0.0602(7)$ & $30.902(9)$ & $0.339(5)$ & $0.0580(9)$ \\
\hline 1.50 & $20.313(9)$ & $0.431(4)$ & $0.0688(6)$ & 28.704(9) & $0.418(9)$ & $0.0667(14)$ \\
\hline 1.60 & $19.049(10)$ & $0.514(9)$ & $0.0768(13)$ & $26.867(11)$ & $0.503(7)$ & $0.0752(10)$ \\
\hline
\end{tabular}



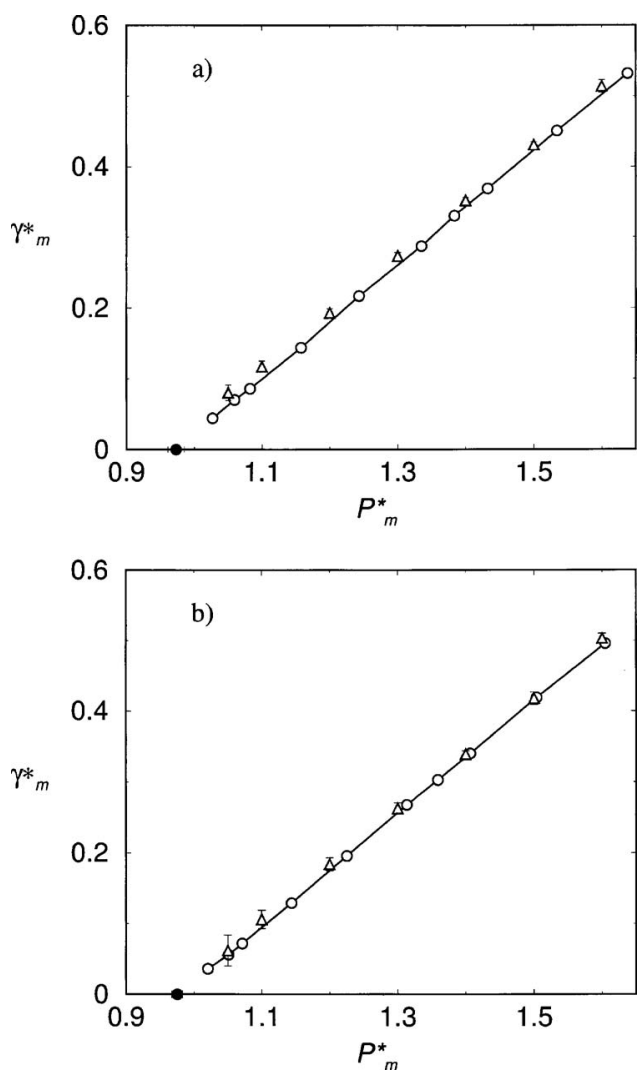

FIG. 4. The reduced surface tension $\gamma_{m}^{*}=\beta \gamma_{m} \sigma^{2}$ of the WR mixture as a function of the reduced pressure $P_{m}^{*}=\beta P_{m} \sigma^{3}$. The data are obtained from direct simulation of the fluid-fluid interface for systems of (a) $N=1500$ and (b) $N=4000$ particles. Data are included for simulations performed at constant volume (circles) and at constant pressure (triangles). The filled symbols represent the critical point.

In order to get a quantitative insight into the dependence of the interfacial tension with the area of the interface $A=L^{2}$, we also undertake a series of simulations of the WR mixture with the transverse dimension $L$ varying in the range $4 \leqslant L / \sigma \leqslant 10$. The simulations are run at a constant pressure $P_{m}^{*}=1.5$, and the total number of particles is chosen such that $N=32(L / \sigma)^{2}$. The behavior of $\gamma$ with $L$ is expected to be of the form ${ }^{26,28}$

$$
\gamma_{L}=\gamma_{\infty}+\frac{B}{L^{2}}+\frac{C \ln L}{L^{2}},
$$

where $\gamma_{L}$ is the surface tension of the finite-size system, $\gamma_{\infty}$ is the corresponding value in the thermodynamic limit, and $B$ and $C$ are (unknown) constants. Following the procedure described in Ref. 26, we omit the last term in Eq. (23) and extrapolate the simulation data for different system sizes using the single extrapolating variable $1 / L^{2}$. For a reduced pressure of $P_{m}^{*}=1.5$, we find $B / \sigma^{2} \simeq 1.566$ and $\gamma_{\infty}^{*}$ $=0.403(4)$. The latter value is to be compared with the values $0.431(4)$ and $0.418(9)$ obtained for systems containing $N$ $=1500(L / \sigma=7.368)$ and $N=4000(L / \sigma=10)$ particles, respectively (see Table III). Our values of the interfacial tension appear to be consistent with (but slightly lower than) those obtained by Malijevský and Sokolowski ${ }^{20}$ in their recent study. An additional series of simulations are performed to analyze any possible dependence of the computed values
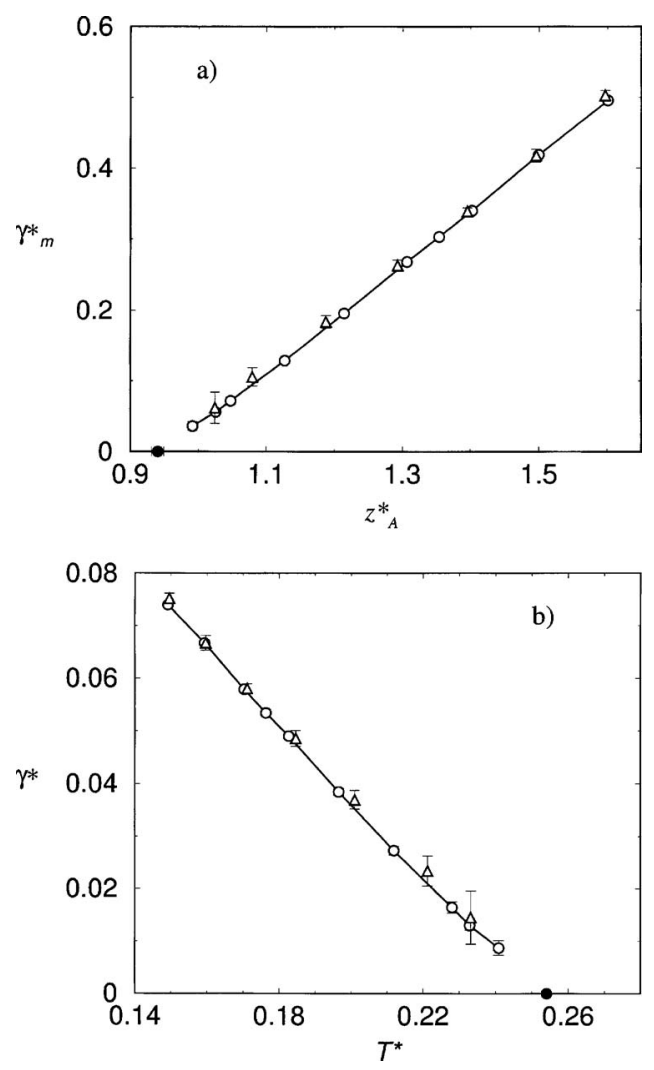

FIG. 5. (a) The reduced surface tension $\gamma_{m}^{*}=\beta \gamma_{m} \sigma^{2}$ of the WR mixture as a function of the activity $z_{A}^{*}=\exp \left(\beta \tilde{\mu}_{A}\right)$ of component $A$ of the mixture as obtained from constant volume (circles) and constant pressure (triangles) simulations of the fluid-fluid interface for systems of $N=4000$ particles. (b) The corresponding vapor-liquid surface tension $\gamma^{*}=\gamma \sigma^{2} / \varepsilon$ of the penetrable sphere model as a function of the reduced temperature $T^{*}=k_{B} T / \varepsilon$. The filled symbols represent the critical point.

of the interfacial tension on the longitudinal dimension of the simulation cell $L_{z}$. The simulations are carried out at constant pressure of $P_{m}^{*}=1.5$ and a fixed surface area $A=L \times L$ with $L=10 \sigma$ for varying numbers of particles ranging from $N=2000$ (average $L_{z} \simeq 15.15 \sigma$ ) to $N=4000$ (average $L_{z} \simeq 28.74 \sigma$ ). No particular dependence of $\gamma$ on $L_{z}$ is found for the systems considered here within statistical accuracy of the simulation results.

The variation of the interfacial tension of the WR mixture with the relative activity $z_{A}^{*}$ is shown in Fig. 5. The surface tension of the vapor-liquid interface of the isomorphous one-component penetrable sphere model is determined from Eq. (13). The corresponding values are included in Tables II and III and are shown in Fig. 5 in terms of the reduced temperature, where the latter follows from the transcription relation (10).

The asymptotic behavior of the surface tension in the neighborhood of the vapor-liquid critical point is known to be given ${ }^{28,49}$ by $\gamma \propto|t|^{2 \nu}$, where $2 \nu \approx 1.26$ is the corresponding critical exponent. According to Eq. (15), the appropriate asymptotic relations for the interfacial tension $\gamma_{m}$ of the athermal WR mixture are thus expected to be given by

$$
\gamma_{m} \propto|\bar{z}|^{2 \nu}, \quad \gamma_{m} \propto\left|\rho_{A}-\rho_{B}\right|^{2 \nu / \beta}, \quad \gamma_{m} \propto\left(\rho-\rho_{c}\right)^{2 \nu /(1-\alpha)} .
$$

The direct simulation of the inhomogeneous system, however, is not the most appropriate technique to probe the criti- 
cal region; the width of the interface diverges as the system approaches the critical point and the interface exhibits increasingly sluggish fluctuations. A full analysis of the behavior of the surface tension in this region would instead require the application of more sophisticated simulation approaches involving finite-size scaling techniques in combination with appropriate algorithms that alleviate slowing-down effects expected in the critical region. The analysis of the critical region is out of the scope of this work and is not undertaken here.

\section{CONCLUSIONS}

In this work we address the major issues involving the simulation of the surface tension associated with the fluidfluid interface of the Widom-Rowlinson binary mixture. The fluid-fluid coexistence properties are examined first by performing simulations in the semigrand canonical ensemble at constant volume or at constant pressure. We pay particular attention to the dependence of the coexistence properties on the pressure. This magnitude is usually ignored in related studies of athermal mixtures. For systems characterized by hard interactions (such as the WR mixture) the pressure can be easily obtained in constant-volume simulations by computing the average number of molecular overlaps that occur in the system under a virtual decrease of the volume of the system. The results obtained from constant-volume and constant-pressure simulations are seen to be fully consistent, and they do not exhibit any size dependence for the range of thermodynamic parameters considered here. The use of the appropriate transcription relations allows us to examine the vapor-liquid coexistence properties of the equivalent onecomponent penetrable sphere model from the fluid-fluid coexistence properties of the WR mixture. The extrapolation of the coexistence properties to the critical region by using the appropriate asymptotic relations provides rough estimates of the thermodynamic properties of the critical point. These are found to be fully consistent with those obtained from a more rigorous analysis based on finite-size scaling techniques.

As for the calculation of the surface tension, we follow the standard virial (mechanical) route that relates the interfacial tension with the relevant components of the pressure tensor in a direct simulation involving the coexisting fluid ( $A$-rich and $B$-rich) phases. This approach is appropriate for an accurate computation of the components of the pressure tensor. This is indeed the case in our simulations, in which both components are determined with a large degree of accuracy. For constant-volume simulations, the (microscopically averaged) normal and tangential tensorial components are determined from the average number of molecular overlaps under virtual volume changes that decrease the longitudinal and transverse dimensions of the simulation cell, respectively. In the case of constant-pressure simulations, the normal pressure is fixed from the outset and one need only calculate the average tangential component. Both routes are found to yield consistent values of the surface tension as long as the system (or, more precisely, the interfacial area) is sufficiently large. The dependence of the surface tension on the system size is examined for a single state point, from which we infer that the computed values of the surface tension for the largest systems considered here overestimate the values in the thermodynamic limit by about $3 \%$. As expected, we find that the values of surface tension are more sensitive to the tangential (relative to the interface) dimension of the simulation than they are to the longitudinal dimension (provided the latter is large enough so as to prevent direct interactions of the two interfaces). Our approach is clearly not appropriate to gauge the surface tension in the vicinity of the critical region, but can be used with confidence over a range of conditions far from the critical region. More sophisticated techniques based on a finite-size scaling analysis are clearly better suited for studies of the critical behavior.

\section{ACKNOWLEDGMENTS}

Financial support is due to the Spanish Dirección General de Investigación [Project Nos. FIS2004-06627-C02-01 (E.d.M.) and FIS2004-02954-C03-01 (N.G.A.)] and from Universidad de Huelva and Junta de Andalucía. Additional funding from the Dirección General de Universidades e Investigación (Comunidad de Madrid, Spain) under the MOSSNOHO-CM program (Grant No. S0505/ESP/0299) and from the Engineering and Physical Sciences EPSRC of the UK (Grant Nos. GR/N20317, GR/N03358, GR/N35991, GR/R09497, and EP/E016340), the Joint Research Equipment Initiative (JREI) (GR/M94427), and the Royal SocietyWolfson Foundation refurbishment grant is also acknowledged. Finally we are grateful to the Royal Society for the award of a International Short Visit grant which has facilitated the collaborative work.

${ }^{1}$ J. M. Brader, R. Evans, and M. Schmidt, Mol. Phys. 101, 3349 (2003).

${ }^{2}$ L. Harnau and S. Dietrich, in Soft Matter, edited by G. Gompper and M. Schick (Wiley-VCH, Berlin, 2006), Vol. 3.

${ }^{3}$ B. Widom and J. S. Rowlinson, J. Chem. Phys. 52, 1670 (1970).

${ }^{4}$ K. Jagannathan and A. Yethiraj, J. Chem. Phys. 118, 7907 (2003).

${ }^{5}$ W. T. Góźdź, J. Chem. Phys. 119, 3309 (2003).

${ }^{6}$ J. S. Rowlinson and B. Widom, Molecular Theory of Capillarity (Clarendon, Oxford, 1982).

${ }^{7}$ C. A. Leng, J. S. Rowlinson, and S. M. Thompson, Proc. R. Soc. London, Ser. A 352, 1 (1976).

${ }^{8}$ C. A. Leng, J. S. Rowlinson, and S. M. Thompson, Proc. R. Soc. London, Ser. A 358, 267 (1978).

${ }^{9}$ J. M. Harrington and J. S. Rowlinson, Proc. R. Soc. London, Ser. A 367, 15 (1979)

${ }^{10}$ J. P. Straley, M. A. Cotter, T.-J. Lie, and B. Widom, J. Chem. Phys. 57, 4484 (1972)

${ }^{11}$ P. Borgelt, C. Hoheisel, and G. Stell, J. Chem. Phys. 92, 6161 (1990).

${ }^{12}$ C.-Y. Shew and A. Yethiraj, J. Chem. Phys. 104, 7665 (1996).

${ }^{13}$ Al. Malijevský, S. Sokolowski, and T. Zientarski, J. Chem. Phys. 125, 114505 (2006).

${ }^{14}$ J. M. Brader and R. L. C. Vink, J. Phys.: Condens. Matter 19, 036101 (2007).

${ }^{15}$ M. Schmidt, Phys. Rev. E 63, 010101 (2000).

${ }^{16}$ G. Johnson, H. Gould, J. Machta, and L. K. Chayes, Phys. Rev. Lett. 79, 2612 (1997).

${ }^{17}$ A. Buhot, J. Chem. Phys. 122, 024105 (2005).

${ }^{18}$ K. Jagannathan and A. Yethiraj, J. Chem. Phys. 122, 244506 (2005).

${ }^{19}$ R. L. C. Vink, J. Chem. Phys. 124, 094502 (2006).

${ }^{20}$ Al. Malijevský and S. Sokolowski, J. Chem. Phys. 126, 106101 (2007).

${ }^{21}$ Y. Duda, E. Vakarin, and J. Alejandre, J. Colloid Interface Sci. 258, 10 (2003).

${ }^{22}$ R. L. C. Vink and J. Horbach, J. Chem. Phys. 121, 3253 (2004).

${ }^{23}$ R. L. C. Vink and J. Horbach, J. Phys.: Condens. Matter 16, S3807 
(2004).

${ }^{24}$ R. L. C. Vink and M. Schmidt, Phys. Rev. E 71, 051406 (2005).

${ }^{25}$ A. Fortini, M. Dijkstra, M. Schmidt, and P. P. F. Wessels, Phys. Rev. E 71, 051403 (2005).

${ }^{26}$ R. L. C. Vink, J. Horbach, and K. Binder, Phys. Rev. E 71, 011401 (2005).

${ }^{27}$ G. J. Gloor, G. Jackson, F. J. Blas, and E. de Miguel, J. Chem. Phys. 123, 134703 (2005).

${ }^{28}$ K. Binder, Phys. Rev. A 25, 1699 (1982).

${ }^{29}$ J. L. Potoff and A. T. Panagiotopoulos, J. Chem. Phys. 112, 6411 (2000).

${ }^{30}$ E. Salomons and M. Marechal, J. Phys.: Condens. Matter 3, 3645 (1991).

${ }^{31}$ E. Díaz-Herrera, J. Alejandre, G. Ramírez-Santiago, and F. Forstmann, J. Chem. Phys. 110, 8084 (1999).

${ }^{32}$ A. Mejía, J. C. Pamies, D. Duque, H. Segura, and L. F. Vega, J. Chem. Phys. 123, 034505 (2005).

${ }^{33}$ Y. Zhang, S. E. Feller, B. R. Brooks, and R. W. Pastor, J. Chem. Phys. 103, 10252 (1995).

${ }^{34}$ S. Senapati and M. L. Berkowitz, Phys. Rev. Lett. 87, 176101 (2001).

${ }^{35}$ D. A. Kofke and E. D. Glandt, Mol. Phys. 64, 1105 (1988).
${ }^{36}$ H.-P. Deutsch and K. Binder, Macromolecules 25, 6214 (1992).

${ }^{37}$ E. de Miguel, E. Martín del Río, and M. M. Telo da Gama, J. Chem. Phys. 103, 6188 (1995).

${ }^{38}$ E. Lomba, M. Álvarez, L. L. Lee, and N. G. Almarza, J. Chem. Phys. 104, 4180 (1996).

${ }^{39}$ J. P. Hansen and I. R. McDonald, Theory of Simple Liquids, 3rd ed. (Academic, London, 2006).

${ }^{40}$ R. Eppenga and D. Frenkel, Mol. Phys. 52, 1303 (1984).

${ }^{41}$ E. de Miguel and M. P. Allen, Mol. Phys. 76, 1275 (1992).

${ }^{42}$ E. de Miguel and E. Martín del Río, J. Chem. Phys. 115, 9072 (2001).

${ }^{43}$ M. P. Allen, J. Chem. Phys. 124, 214103 (2006).

${ }^{44}$ E. de Miguel and G. Jackson, Mol. Phys. 104, 3717 (2006).

${ }^{45}$ B. Widom, J. Chem. Phys. 39, 2808 (1963).

${ }^{46}$ J. M. Haile, Molecular Dynamics Simulation (Wiley, New York, 1992).

${ }^{47}$ E. de Miguel and G. Jackson, J. Chem. Phys. 125, 164109 (2006).

${ }^{48}$ D. Frenkel and B. Smit, Understanding Molecular Simulation (Academic, New York, 1996), p. 108.

${ }^{49}$ B. Widom, in Phase Transitions and Critical Phenomena edited by C. Domb and M. S. Green (Wiley, New York, 1972), Vol. 2, p. 79. 